\title{
Exact solutions for fractional DDEs via auxiliary equation method coupled with the fractional complex transform
}

\author{
İsmail Aslan ${ }^{* \dagger}$
}

\section{Communicated by C. Cuevas}

Dynamical behavior of many nonlinear systems can be described by fractional-order equations. This study is devoted to fractional differential-difference equations of rational type. Our focus is on the construction of exact solutions by means of the (G'/G)-expansion method coupled with the so-called fractional complex transform. The solution procedure is elucidated through two generalized time-fractional differential-difference equations of rational type. As a result, three types of discrete solutions emerged: hyperbolic, trigonometric, and rational. Copyright @ 2016 John Wiley \& Sons, Ltd.

Keywords: differential-difference equation; fractional calculus; local fractional derivative; (G'/G)-expansion method

\section{Introduction}

Differential-difference equations (DDEs), or lattice equations, are used in the chemical, biological, physical, engineering, social, and medical sciences. Hence, their analysis plays a very important role in the applied sciences. Starting from pioneering ideas going back to Fermi, Pasta, and Ulam [1], many DDEs have been developed: for instance, discrete KdV equation [2], Toda lattice equation [3], Volterra lattice equation [4], Ablowitz-Ladik lattice equation [5], discrete modified KdV equation [6], discrete sine-Gordon equation [7], and so forth. These DDEs commonly appear in the form $d u_{n} / d t=P\left(u_{n-1}, u_{n}, u_{n+1}\right)$ where $n \in Z, P$ is a polynomial function of its arguments, $u_{n}(t)=u(n, t)$ denotes the displacement of the $n$th particle from the equilibrium position. Besides their physical relevance, DDEs also play an important role in numerical simulations of nonlinear PDEs. Lately, He et al. [8] suggested the extension of fractional calculus to DDEs when pointing out new directions in nonlinear science. In fact, in recent years, many nonlinear equations in science and engineering have been generalized to non-integer orders so as to provide new models. It can be observed that most of the applications of fractional calculus amount to replacing the time derivative in a nonlinear equation with a fractional order derivative. Thus, we believe that a beneficial generalization of DDEs to a fractional order is possible and important.

Indeed, being invented by Newton and Leibniz over three centuries ago, fractional calculus is a generalization of the ordinary differentiation and integration to non-integer order. Using integer-order models for complex natural (or man-made) systems is simply for our own convenience while the nature runs in a fractional order dynamical way. In recent decades, a rapid development in the theory of fractional differential equations (FDEs) has been witnessed due to their exact description of many real-life problems such as signal processing, control problem, polymers, non-Brownian motion, viscoelastic materials, finance, and fractional dynamics (Kilbas et al. [9]). Hence, finding exact/approximate solutions to FDEs has become an important task. There are various analytical methods for fractional calculus, among them are the homotopy analysis method [10], the discrete method [11], the tau approach [12], the finite difference method [13], the B-spline functions method [14], the ( $\left.G^{\prime} / G\right)$-expansion method [15], the operational matrix method [16], the finite element method [17], the variational methods [18], the homotopy perturbation method [19], the fractional sub-equation method [20], the first integral method [21], and the others [22-25].

In this study, we particularly focus on fractional DDEs of rational type $d /{ }^{\alpha} u_{n} / d t^{\alpha}=R\left(u_{n-1}, u_{n}, u_{n+1}\right), 0<\alpha \leq 1$, where $d^{\alpha} u_{n} / d t^{\alpha}$ denotes a local fractional derivative (in time) of $\operatorname{order} \alpha, R$ is a polynomial function of its arguments and $n \in Z$. As far as we could verify, DDEs of such types received relatively little attention. To achieve our goal, we consider the following time-fractional DDEs

\footnotetext{
Department of Mathematics, Izmir Institute of Technology, Urla, Izmir 35430, Turkey

* Correspondence to: Ismail Aslan, Department of Mathematics, Izmir Institute of Technology, Urla, Izmir 35430, Turkey.

† E-mail: ismailaslan@iyte.edu.tr
} 


$$
\begin{gathered}
\frac{d^{\alpha} u_{n}}{d t^{\alpha}}=\frac{u_{n-1}-u_{n+1}}{1-u_{n-1}+u_{n+1}}, 0<\alpha \leq 1, \\
\frac{d^{\alpha} u_{n}}{d t^{\alpha}}=\frac{4\left(u_{n-1}-u_{n+1}\right) u_{n}^{2}\left(1-u_{n}^{2}\right)}{\left(u_{n-1}+u_{n}\right)\left(u_{n}+u_{n+1}\right)}, 0<\alpha \leq 1,
\end{gathered}
$$

which are proposed in [26,27]. Equation (1) and Equation (2) are related to the Volterra equation and discrete modified KdV equation through dependent variable transformations, respectively. Some applications of fractional DDEs can be seen in the studies [28-31] where the fractional derivatives in the sense of modified Riemann-Liouville derivative and the basic (G'/G)-expansion method [32,33] were employed for constructing exact solutions. The advantage of the $\left(G^{\prime} / G\right)$-expansion method is that one treats nonlinear problems by essentially linear methods. The method is based on the explicit linearization of nonlinear equations for traveling waves with a certain substitution, which leads to a second-order differential equation with constant coefficients. The (G'/G)-expansion method provides not only more general forms of solutions but also periodic and solitary waves. Besides, it handles nonlinear equations in a direct manner with no requirement for initial/boundary condition or initial trial function at the outset. The solution procedure, using a computer algebra system like Mathematica, is of utter simplicity.

The rest of this paper is organized as follows: In Section 2, we describe the local fractional derivative and its properties. In Section 3, we introduce the fractional complex transform and explain the conversion of FDEs into ordinary differential equations (ODEs). In Section 4, an adaptation of the basic ( $\left.G^{\prime} / G\right)$-expansion method for solving DDEs is summarized. In Sections 5 and 6, special attention is given to two time-fractional differential-difference equations of rational type coupled with the local fractional derivative. Finally, concluding remarks are given in Section 7.

\section{Local fractional derivative}

There are many different types of fractional derivatives. For instance, Ortigueira and Machado [34] analyzed several important definitions, namely, the Grünwald-Letnikov, Riemann-Liouville, Liouville-Caputo derivatives and the Riesz potential. Another systematical study is given by Yang [35, 36]. Moreover, He [37] introduced the basic properties of some fractional derivatives by the variational iteration method. Several local versions of fractional derivatives have been proposed for the investigation of local behavior of fractional models. For example, Cresson's derivative [38], Kolwankar-Gangal local fractional derivative [39], and Jumarie's modified RiemannLiouville derivative [40]. Indeed, Liouville-Caputo derivatives are defined only for differentiable functions, while $f$ can be a continuous (but not necessarily differentiable) function. The Riemann-Liouville definition is suitable for any functions that are continuous but not differentiable anywhere; however, $D_{x}^{\alpha}(f(x)) \neq 0$ when $f(x)$ is a constant. To overcome the pitfalls, Jumarie [40] suggested a modification of the Riemann-Liouville fractional derivative where $f$ is a continuous (but not necessarily differentiable) function. However, the chain rule for those derivatives is complicated. The local fractional derivative of $f(x)$ of order $\alpha(0<\alpha \leq 1)$ at $x=x_{0}$ is defined as

$$
f^{(\alpha)}\left(x_{0}\right)=\left.\frac{d^{\alpha} f(x)}{d x^{\alpha}}\right|_{x=x_{0}}=\lim _{x \rightarrow x_{0}} \frac{\Delta^{\alpha}\left(f(x)-f\left(x_{0}\right)\right)}{\left(x-x_{0}\right)^{\alpha}},
$$

where $\Delta^{\alpha}\left(f(x)-f\left(x_{0}\right)\right) \cong \Gamma(1+\alpha)\left(f(x)-f\left(x_{0}\right)\right)$. The local fractional derivative (3) has the following properties:

$$
\begin{gathered}
\frac{d^{\alpha} c}{d x^{\alpha}}=0, \frac{d^{\alpha}}{d x^{\alpha}}[c f]=c \frac{d^{\alpha} f}{d x^{\alpha}}, \frac{d^{\alpha} x^{\beta}}{d x^{\alpha}}=\frac{\Gamma(1+\beta)}{\Gamma(1+\beta-\alpha)} x^{\beta-\alpha}, \beta \geq \alpha>0 . \\
\frac{d^{k \alpha} f(x)}{d x^{k \alpha}}=\overbrace{\frac{d^{\alpha}}{d x^{\alpha}} \cdots \frac{d^{\alpha}}{d x^{\alpha}}}^{\text {ktimes }} f(x), \frac{d^{\alpha} f(g(x))}{d x^{\alpha}}=f^{(1)}(g(x)) g^{(\alpha)}(x)=f^{(\alpha)}(g(x)) g^{(1)}(x) .
\end{gathered}
$$

Recently, the local fractional derivative (3) has attracted much attention due to its simple chain rule. By the same token, the local fractional partial derivative of $f(x, t)$ of order $\alpha(0<\alpha \leq 1)$ at $\left(x_{0}, t\right)$ is defined as

$$
\frac{\partial^{\alpha} f\left(x_{0}, t\right)}{\partial x^{\alpha}}=\lim _{x \rightarrow x_{0}} \frac{\Delta^{\alpha}\left(f(x, t)-f\left(x_{0}, t\right)\right)}{\left(x-x_{0}\right)^{\alpha}} \text {, where } \Delta^{\alpha}\left(f(x, t)-f\left(x_{0}, t\right)\right) \cong \Gamma(1+\alpha)\left(f(x, t)-f\left(x_{0}, t\right)\right) \text {. }
$$

\section{Fractional complex transform}

The fractional complex transform was first proposed in 2010 by Li and He [41] to convert FDEs into ODEs so that all analytical methods for advanced calculus can be easily applied to fractional calculus. This approach requires no special knowledge of fractional calculus. For example, consider a fractional nonlinear wave equation [37] in the form

$$
\frac{\partial^{2 \alpha} u}{\partial t^{2 \alpha}}-\frac{\partial^{2 \beta} u}{\partial x^{2 \beta}}+u-u^{3}=0,0<\alpha, \beta<1,
$$


where $\partial^{\alpha} u / \partial t^{\alpha}$ is the local fractional partial derivative. By means of the fractional complex transform

$$
T=\frac{t^{\alpha}}{\Gamma(1+\alpha)}, X=\frac{x^{\beta}}{\Gamma(1+\beta)},
$$

Equation (6) can be converted to its differential partner that reads

$$
\frac{\partial^{2} u}{\partial T^{2}}-\frac{\partial^{2} u}{\partial X^{2}}+u-u^{3}=0
$$

Using a wave transformation of the form $\xi=k X+w T$, where $k$ and $w$ are constants, the Equation (8) reduces to the following ODE

$$
\left(w^{2}-k^{2}\right) u^{\prime \prime}+u-u^{3}=0
$$

\section{Methodology}

To reveal the basic idea of our method, we consider a system of $M$ fractional DDEs in the form

$$
\Omega\left(u_{n+p_{1}}(x), \ldots, u_{n+p_{k}}(x), \ldots, u_{n+p_{1}}^{\alpha}(x), \ldots, u_{n+p_{k}}^{\alpha}(x), \ldots, u_{n+p_{1}}^{(r \alpha)}(x), \ldots, u_{n+p_{k}}^{(r \alpha)}(x)\right)=0,0<\alpha \leq 1
$$

where the dependent variable $u_{n}$ have $M$ components $u_{i, n}$ and so do its shifts; the continuous variable $\mathrm{x}$ has $N$ components $x_{i}$; the discrete variable $\mathrm{n}$ has $Q$ components $n_{j}$; the $k$ shift vectors $\mathrm{p}_{i} \in Z^{Q}$; and $\mathrm{u}^{(r \alpha)}(\mathrm{x})$ denotes the collection of fractional derivative terms of order $r \alpha$. To search for exact solutions of Equation (10), we first take the fractional complex transformation

$$
\mathrm{u}_{\mathrm{n}+\mathrm{p}_{\mathrm{s}}}(\mathrm{x})=\mathrm{U}_{\mathrm{n}+\mathrm{p}_{\mathrm{s}}}\left(\xi_{\mathrm{n}}\right), \xi_{\mathrm{n}}=\sum_{i=1}^{Q} d_{i} n_{i}+\sum_{j=1}^{N} \frac{c_{j}}{\Gamma(1+\alpha)} x_{j}^{\alpha}+\zeta_{,}(s=1,2, \ldots, k),
$$

into consideration where the coefficients $c_{1}, c_{2}, \ldots, c_{N}, d_{1}, d_{2}, \ldots, d_{Q}$ and the phase $\zeta$ are all constants, while the symbol $\Gamma$ denotes the gamma function. Then, Equation (10) changes into a system of integer order in the form

$$
\Omega\left(U_{n+p_{1}}\left(\xi_{n}\right), \ldots, U_{n+p_{k}}\left(\xi_{n}\right), \ldots, U_{n+p_{1}}^{\prime}\left(\xi_{n}\right), \ldots, U_{n+p_{k}}^{\prime}\left(\xi_{n}\right), \ldots, U_{n+p_{1}}^{(r)}\left(\xi_{n}\right), \ldots, U_{n+p_{k}}^{(r)}\left(\xi_{n}\right)\right)=0
$$

To obtain an exact solution for (12), a finite expansion in $\frac{G^{\prime}\left(\xi_{n}\right)}{G\left(\xi_{n}\right)}$ like

$$
\mathrm{U}_{\mathrm{n}}\left(\xi_{\mathrm{n}}\right)=\sum_{l=0}^{m} a_{l}\left(\frac{G^{\prime}\left(\xi_{\mathrm{n}}\right)}{G\left(\xi_{\mathrm{n}}\right)}\right)^{l}, a_{m} \neq 0
$$

is proposed, where $m$ is a positive integer, $a_{i}$ 's are constants to be determined, $G\left(\xi_{n}\right)$ is a solution of the second-order linear ordinary differential equation

$$
G^{\prime \prime}\left(\xi_{\mathrm{n}}\right)+\lambda G^{\prime}\left(\xi_{\mathrm{n}}\right)+\mu G\left(\xi_{\mathrm{n}}\right)=0,
$$

in which $\lambda$ and $\mu$ are arbitrary parameters and prime denotes derivative with respect to $\xi_{\mathrm{n}}$. The general solution of Equation (14) is well known to us. We have three cases: (i) if $\lambda^{2}-4 \mu>0$, then Equation (14) admits solutions in terms of hyperbolic functions; (ii) if $\lambda^{2}-4 \mu<0$, then Equation (14) admits solutions in terms of trigonometric functions; and (iii) if $\lambda^{2}-4 \mu=0$, then Equation (14) admits rational solutions, see [42] for the more details of the procedure.

\section{Exact solutions for Equation (1)}

We first introduce the fractional complex transformation

$$
u_{n}=U_{n}\left(\xi_{n}\right), \xi_{n}=d n+\frac{k}{\Gamma(1+\alpha)} t^{\alpha}+\chi_{1}
$$

where $d$ and $k$ are real parameters to be specified, while $\chi$ denotes the phase shift. Substituting (15) into Equation (1) gives

$$
k U_{n}^{\prime}\left(1-U_{n-1}+U_{n+1}\right)-\left(U_{n-1}-U_{n+1}\right)=0,
$$


where prime denotes ordinary derivative with respect to the new independent variable $\xi_{n}$. Our procedure suggests then to look for special solutions of (16) in the form

$$
U_{n}=a_{0}+a_{1}\left(\frac{G^{\prime}}{G}\right), a_{1} \neq 0
$$

where $G=G\left(\xi_{n}\right)$ satisfies Equation (14), while $a_{0}$ and $a_{1}$ are arbitrary constants to be determined at the stage of solving the problem.

\subsection{Hyperbolic function solutions}

In case $\mu<0$, we get a relation among the parameters as follows

$$
\lambda=0, a_{0}=a_{0}, a_{1}=-\frac{\tanh (d \sqrt{-\mu})}{2 \sqrt{-\mu}}, k=-\frac{\sinh (2 d \sqrt{-\mu})}{\sqrt{-\mu}},
$$

which yields a hyperbolic function solution to Equation (1) as

$$
u_{n}(t)=a_{0}-\frac{1}{2} \tanh (d \sqrt{-\mu})\left(\frac{C_{1} \cosh \left(\sqrt{-\mu} \xi_{n}\right)+C_{2} \sinh \left(\sqrt{-\mu} \xi_{n}\right)}{C_{1} \sinh \left(\sqrt{-\mu} \xi_{n}\right)+C_{2} \cosh \left(\sqrt{-\mu} \xi_{n}\right)}\right)
$$

where $\xi_{n}=d n-\frac{\sinh (2 d \sqrt{-\mu})}{\Gamma(1+\alpha) \sqrt{-\mu}} t^{\alpha}+\chi$, while $a_{0}, d, \chi, \mu(<0), C_{1}$ and $C_{2}$ remain arbitrary.

\subsection{Trigonometric function solutions}

In case $\mu>0$, we get a relation among the parameters as follows

$$
\lambda=0, a_{0}=a_{0}, a_{1}=-\frac{\tan (d \sqrt{\mu})}{2 \sqrt{\mu}}, k=-\frac{\sin (2 d \sqrt{\mu})}{\sqrt{\mu}},
$$

which gives a trigonometric function solution to Equation (1) as

$$
u_{n}(t)=a_{0}-\frac{1}{2} \tan (d \sqrt{\mu})\left(\frac{-C_{1} \sin \left(\sqrt{\mu} \xi_{n}\right)+C_{2} \cos \left(\sqrt{\mu} \xi_{n}\right)}{C_{1} \cos \left(\sqrt{\mu} \xi_{n}\right)+C_{2} \sin \left(\sqrt{\mu} \xi_{n}\right)}\right)
$$

where $\xi_{n}=d n-\frac{\sin (2 d \sqrt{\mu})}{\Gamma(1+\alpha) \sqrt{\mu}} t^{\alpha}+\chi$, while $a_{0}, d, \chi, \mu(>0), C_{1}$ and $C_{2}$ remain arbitrary.

\subsection{Rational function solutions}

In case $\mu=0$, we get a relation among the parameters as follows

$$
\lambda=0, a_{0}=a_{0}, a_{1}=-\frac{d}{2}, k=-2 d,
$$

which yields a rational function solution to Equation (1) as

$$
u_{n}(t)=a_{0}+\frac{d}{2}\left(\frac{C_{1}}{C_{1}\left(d n-\frac{2 d}{\Gamma(1+\alpha)} t^{\alpha}+\chi\right)+C_{2}}\right)
$$

where $a_{0}, d, \chi, C_{1}$, and $C_{2}$ remain arbitrary.

Remark 1

If we set ' $\mu=-1$ and $C_{1}=0$ ' or ' $C_{2}=0$ and $\mu=-1$ ', respectively, the solution (19) becomes

$$
\begin{aligned}
& u_{n}(t)=a_{0}-\frac{1}{2} \tanh (d) \tanh \left(d n-\frac{\sinh (2 d)}{\Gamma(1+\alpha)} t^{\alpha}+\chi\right), \\
& u_{n}(t)=a_{0}-\frac{1}{2} \tanh (d) \operatorname{coth}\left(d n-\frac{\sinh (2 d)}{\Gamma(1+\alpha)} t^{\alpha}+\chi\right),
\end{aligned}
$$
wave solution. 
By the same token, setting ' $\mu=1$ and $C_{2}=0^{\prime}$ or ' $C_{1}=0$ and $\mu=1$ ', respectively, the solution (21) becomes singular periodic wave solutions in the form

$$
\begin{aligned}
& u_{n}(t)=a_{0}+\frac{1}{2} \tan (d) \tan \left(d n-\frac{\sin (2 d)}{\Gamma(1+\alpha)} t^{\alpha}+\chi\right) \\
& u_{n}(t)=a_{0}-\frac{1}{2} \tan (d) \cot \left(d n-\frac{\sin (2 d)}{\Gamma(1+\alpha)} t^{\alpha}+\chi\right)
\end{aligned}
$$

where $a_{0}, d$ and $\chi$ remain arbitrary.

\section{Exact solutions for Equation (2)}

Like before, we first make the fractional complex transformation

$$
u_{n}=U_{n}\left(\xi_{n}\right), \quad \xi_{n}=d n+\frac{k}{\Gamma(1+\alpha)} t^{\alpha}+\chi_{1}
$$

where $d$ and $k$ are real parameters to be specified, while $\chi$ denotes the phase shift. Substituting (28) into Equation (2) leads to the equation

$$
k U_{n}^{\prime}\left(U_{n-1}+U_{n}\right)\left(U_{n}+U_{n+1}\right)-4\left(U_{n-1}-U_{n+1}\right) U_{n}^{2}\left(1-U_{n}^{2}\right)=0,
$$

where prime denotes ordinary derivative with respect to the new independent variable $\xi_{n}$. Our procedure suggests then to look for special solutions of the system (29) in the form

$$
U_{n}=a_{0}+a_{1}\left(\frac{G^{\prime}}{G}\right), a_{1} \neq 0
$$

where $G=G\left(\xi_{n}\right)$ satisfies Equation (14), while $a_{0}$ and $a_{1}$ are arbitrary constants to be specified.

\subsection{Hyperbolic function solutions}

In case $\mu<0$, we get relations among the parameters as follows

$$
\begin{aligned}
& \lambda=0, a_{0}=-\frac{1}{2} \tanh (d \sqrt{-\mu}), a_{1}= \pm \frac{\tanh (d \sqrt{-\mu})}{2 \sqrt{-\mu}}, k=-\frac{2 \tanh (d \sqrt{-\mu})}{\sqrt{-\mu}} \\
& \lambda=0, a_{0}=\frac{1}{2} \tanh (d \sqrt{-\mu}), a_{1}= \pm \frac{\tanh (d \sqrt{-\mu})}{2 \sqrt{-\mu}}, k=-\frac{2 \tanh (d \sqrt{-\mu})}{\sqrt{-\mu}} .
\end{aligned}
$$

Here and henceforth, the signs are ordered vertically. Setting the parameter values (31) and (32) into the expression (30), one can construct hyperbolic function solutions to Equation (2) as

$$
\begin{aligned}
& u_{n}(t)=-\frac{1}{2} \tanh (d \sqrt{-\mu}) \pm \frac{1}{2} \tanh (d \sqrt{-\mu})\left(\frac{C_{1} \cosh \left(\sqrt{-\mu} \xi_{\mathrm{n}}\right)+C_{2} \sinh \left(\sqrt{-\mu} \xi_{\mathrm{n}}\right)}{C_{1} \sinh \left(\sqrt{-\mu} \xi_{\mathrm{n}}\right)+C_{2} \cosh \left(\sqrt{-\mu} \xi_{\mathrm{n}}\right)}\right) \\
& u_{n}(t)=\frac{1}{2} \tanh (d \sqrt{-\mu}) \pm \frac{1}{2} \tanh (d \sqrt{-\mu})\left(\frac{C_{1} \cosh \left(\sqrt{-\mu} \xi_{\mathrm{n}}\right)+C_{2} \sinh \left(\sqrt{-\mu} \xi_{\mathrm{n}}\right)}{C_{1} \sinh \left(\sqrt{-\mu} \xi_{\mathrm{n}}\right)+C_{2} \cosh \left(\sqrt{-\mu} \xi_{\mathrm{n}}\right)}\right)
\end{aligned}
$$

where $\xi_{n}=d n-\frac{2 \tanh (d \sqrt{-\mu})}{\Gamma(1+\alpha) \sqrt{-\mu}} t^{\alpha}+\chi$, while $d, \chi, \mu(<0), C_{1}$ and $C_{2}$ remain arbitrary.

\subsection{Trigonometric function solutions}

In case $\mu>0$, we get relations among the parameters as follows

$$
\begin{aligned}
& \lambda=0, a_{0}=\mp \frac{1}{2} i \tan (d \sqrt{\mu}), a_{1}=-\frac{\tan (d \sqrt{\mu})}{2 \sqrt{\mu}}, k=-\frac{2 \tan (d \sqrt{\mu})}{\sqrt{\mu}}, \\
& \lambda=0, a_{0}=\mp \frac{1}{2} i \tan (d \sqrt{\mu}), a_{1}=\frac{\tan (d \sqrt{\mu})}{2 \sqrt{\mu}}, k=-\frac{2 \tan (d \sqrt{\mu})}{\sqrt{\mu}} .
\end{aligned}
$$


Setting the parameter values (35) and (36) into the expression (30), one can construct trigonometric function solutions to Equation (1) as

$$
\begin{aligned}
& u_{n}(t)=\mp \frac{1}{2} i \tan (d \sqrt{\mu})-\frac{1}{2} \tan (d \sqrt{\mu})\left(\frac{-C_{1} \sin \left(\sqrt{\mu} \xi_{\mathrm{n}}\right)+C_{2} \cos \left(\sqrt{\mu} \xi_{\mathrm{n}}\right)}{C_{1} \cos \left(\sqrt{\mu} \xi_{\mathrm{n}}\right)+C_{2} \sin \left(\sqrt{\mu} \xi_{\mathrm{n}}\right)}\right), \\
& u_{n}(t)=\mp \frac{1}{2} i \tan (d \sqrt{\mu})+\frac{1}{2} \tan (d \sqrt{\mu})\left(\frac{-C_{1} \sin \left(\sqrt{\mu} \xi_{\mathrm{n}}\right)+C_{2} \cos \left(\sqrt{\mu} \xi_{\mathrm{n}}\right)}{C_{1} \cos \left(\sqrt{\mu} \xi_{\mathrm{n}}\right)+C_{2} \sin \left(\sqrt{\mu} \xi_{\mathrm{n}}\right)}\right),
\end{aligned}
$$

where $\xi_{n}=d n-\frac{2 \tan (d \sqrt{\mu})}{\Gamma(1+\alpha) \sqrt{\mu}} t^{\alpha}+\chi$, while $d, \chi, \mu(>0), C_{1}$ and $C_{2}$ remain arbitrary.

\subsection{Rational function solutions}

In case $\mu=0$, we get a relation among the parameters as follows

$$
\lambda=0, a_{0}=0, a_{1}= \pm \frac{d}{2}, k=-2 d
$$

Inserting the parameter values (39) into the expression (30), one can construct a rational function solution to Equation (2) as

$$
u_{n}(t)= \pm \frac{d}{2}\left(\frac{C_{1}}{C_{1}\left(d n-\frac{2 d}{\Gamma(1+\alpha)} t^{\alpha}+\chi\right)+C_{2}}\right)
$$

where $d(\neq 0), \chi, C_{1}(\neq 0)$ and $C_{2}$ remain arbitrary.

\section{Remark 2}

Of course, as in the preceding section, one can assign special values to the involved arbitrary parameters in the above expressions for further analysis. We skip the procedure for brevity. A close inspection shows that (37) and (38) are complex-valued solutions. Hence, trigonometric function solutions for Equation (2) mathematically exist but they are considered to be physically unrealistic. Naturally, one wonders under what circumstances the corresponding solutions (37) and (38) would have a practical application because of their non-physical nature.

Remark 3

It is an important fact that one should be aware of the limitations of each of the existing methods. There is no guarantee that they will succeed for a specialized nonlinear problem. One of the pitfalls of the $\left(G^{\prime} / G\right)$-expansion method, by assuming the solution of the equation in the polynomial form with many parameters, is that it sometimes leads to inconsistent nonlinear algebraic systems. Another one is that it is entirely algorithmic and involves a large amount of tedious calculations which can become virtually unmanageable if attempted manually.

\section{Conclusion}

Fractional differential equations have received increasing attention during recent years. For example, fractional derivatives provide an excellent instrument for the description of memory and hereditary properties of various materials and processes [43]. However, very little progress has been made to develop the theory of the analogues fractional differential-difference equations. This fact greatly motivated us to use 'fractional-order' derivatives and 'fractional-order' differences in our study. As a result, a newly constructed problem (generalization of classical DDEs to fractional order) is successfully tackled by the basic (G'/G)-expansion method. It is observed that the fractional complex transform makes the solution procedure extremely simple. Such transformation is valid only for general 'wave' solutions for FDEs. We believe that we achieved our goal of gaining exact and explicit solutions for the considered fractional DDEs of rational type, namely, (1) and (2).

\section{Acknowledgements}

The author is grateful to the anonymous referees for careful reading and constructive comments.

\section{References}

1. Fermi E, Pasta J, Ulam S. Collected Papers of Enrico Fermi. University of Chicago Press: Chicago, 1965.

2. Ohta Y, Hirota R. A discrete KdV equation and its Casorati determinant solution. Journal of the Physical Society of Japan 1991; 60:2095.

3. Toda M. Theory of Nonlinear Lattices. Springer-Verlag: New-York, 1989.

4. Wadati M. Transformation theories for nonlinear discrete systems. Prog. Suppl. Theor. Phys 1976; 59:36-63.

5. Ablowitz MJ, Ladik J. Nonlinear differential-difference equation. Journal of Mathematical Physics 1975; 16:598-603.

6. Hirota R. Exact $N$-soliton solution of nonlinear lumped self-dual network equations. Journal of the Physical Society of Japan 1973; 35:289-294.

7. Orfandis SJ. Sine-Gordon equation and nonlinear $\sigma$ model on a lattice. Physical Review D 1978; 18:3828-3832. 
8. He JH, Wu GC, Austin F. The variational iteration method which should be followed. Nonl. Sci. Lett A 2010; 1-30.

9. Kilbas AA, Srivastava HM, Trujillo JJ. Theory and Applications of Fractional Differential Equations. Elsevier: Amsterdam, 2006.

10. Dehghan M, Manafian J, Saadatmandi A. Solving nonlinear fractional partial differential equations using the homotopy analysis method. Numerical Methods Partial Differential Equations 2010; 2(6):448-479.

11. Almeida R, Torres DFM. A discrete method to solve fractional optimal control problems. Nonlinear Dyn 2015; 80:1811-1816.

12. Saadatmandi A, Dehghan M. A tau approach for solution of the space fractional diffusion equation. Computers \& Mathematics with Applications 2011; 6(2):1135-1142.

13. Cui M. Compact finite difference method for the fractional diffusion equation. Journal of Computational Physics 2009; 228:7792-7804.

14. Lakestani M, Dehghan M, Irandoust-pakchin S. The construction of operational matrix of fractional derivatives using B-spline functions. Communications in Nonlinear Science and Numerical Simulation 2012; 1(7):1149-1162.

15. Bin Z. (G'/G)-expansion method for solving fractional partial differential equations in the theory of mathematical physics. Communications in Theoretical Physics 2012; 58:623-630.

16. Saadatmandi A, Dehghan M. A new operational matrix for solving fractional-order differential equations. Computers \& Mathematcs with Applications 2010; 5(9):1326-1336.

17. Huang Q, Huang G, Zhan H. A finite element solution for the fractional advection-dispersion equation. Advances Water Resource 2008; 31:1578-1589.

18. El-Wakil SA, Abulwafa EM, Zahran MA, Mahmoud AA. Time-fractional KdV equation: formulation and solution using variational methods. Nonlinear Dyn 2011; 65:55-63.

19. He JH. A coupling method of a homotopy technique and a perturbation technique for non-linear problems. International Journal of Nonlinear Mech 2000; 35:37-43.

20. Zhang S, Zhang HQ. Fractional sub-equation method and its applications to nonlinear fractional PDEs. Physics Letters A 2011; 375:1069-1073.

21. Lu B. The first integral method for some time fractional differential equations. Journal of Mathematical Analysis and Applications 2012; 395:684-693.

22. Khosravian-Arab H, Dehghan M, Eslahchi MR. Fractional Sturm-Liouville boundary value problems in unbounded domains: Theory and applications. Journal of Computational Physics 2015; 29(9):526-560.

23. Dehghan $M$, Safarpoor $M$, Abbaszadeh $M$. Two high-order numerical algorithms for solving the multi-term time fractional diffusion-wave. Journal of Computational and Applied Mathematics 2011; 290:174-195.

24. Saadatmandi A, Dehghan M. Numerical solution of the higher-order linear Fredholm integro-differential difference equation with variable coefficients. Computers \& Mathematcs with Applications 2010; 59:2996-3004.

25. Dehghan M, Manafian J, Saadatmandi A. Analytical treatment of some partial differential equations arising in mathematical physics by using the Exp-function method. International Journal of Modern Physics B 2011; 25:2965-2981.

26. Narita K. Solutions for the Mikhailov-Shabat-Yamilov difference-differential equations and generalized solutions for the Volterra and the Toda lattice equations. Progress of Theoretical Physics 1998; 99:337-348.

27. Narita K. 1, 2-rational $N$-soliton solutions for difference-differential equations related to the Volterra equation. Chaos Solitons Fract 2002; 13: $1121-1128$.

28. Bekir A, Güner Ö, Ayhan B. Exact solutions of some systems of fractional differential-difference equations. Mathematical Methods in the Applied Sciences 2015; 38:3807-3817.

29. Aslan I, Exact solutions of a fractional-type differential-difference equation related to the discrete MKdV equation, Communications in Theoretical Physics 2014; 61:595-599.

30. Feng QH. Exact solutions for fractional differential-difference equations by an extended Riccati sub-ODE method. Communications in Theoretical Physics 2013; 59:521-527.

31. Aslan I. Symbolic computation of exact solutions for fractional differential-difference equation models. Nonlinear Analysis and Modeling Control; 20:132-144.

32. Wang M, Li X, Zhang J. The (G'/G)-expansion method and travelling wave solutions of nonlinear evolution equations in mathematical physics. Physics Letters A 2008; 372:417-423.

33. Oziş T, Aslan I. Symbolic Computations and exact and explicit solutions of some nonlinear evolution equations in mathematical physics. Communications in Theoretical Physics 2009; 51:577-580.

34. Ortigueira MD, Machado JAT. What is a fractional derivative. Journal of Computational Physics 2015; 293:4-13.

35. Yang XJ. Advanced Local Fractional Calculus and its Applications. World Science Publisher: New York, 2012.

36. Yang XJ. A short note on local fractional calculus of function of one variable. Journal of Applied Library and Information Science 2012; 1:1-13.

37. He JH. A Tutorial review on fractal spacetime and fractional calculus. International Journal of Theoretical Physics 2014; 53:3698-3718.

38. Cresson J. Non-differentiable variational principles. Journal of Mathematical Analysis and Applications 2005; 307:48-64.

39. Kolwankar KM, Gangal AD. Local fractional Fokker-Planck equation. Physical Review Letters 1998; 80:214-217.

40. Jumarie G. Modified Riemann-Liouville derivative and fractional Taylor series of nondifferentiable functions further results. Computers \& Mathematics with Applications 2006; 51:1367-1376.

41. Li ZB, He JH, Mathematical \& Computational Applications. Fractional complex transformation for fractional differential equations 2010; 15:970-973.

42. Aslan I. Analytic solutions to nonlinear differential-difference equations by means of the extended (G'/G) expansion method. Journal of Physics $A$ : Mathematical and Theoretical. 2010; 43:395207.

43. Podlubny I. Fractional Differential Equations, Mathematics in Science and Engineering. Academic Press: San Diego, 1999. 